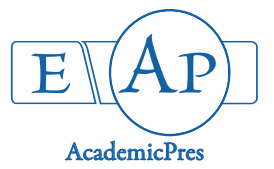

\title{
Prevalence and Antibiogram of Generic Extended-Spectrum $\beta$-Lactam-Resistant Enterobacteria in Healthy Dogs
}

\author{
Madubuike U. ANYANWU ${ }^{1 *}$, Ifeoma C. UGWU ${ }^{1}$, \\ Martins O. EZEKWELU ${ }^{1}$, Obianuju N. OKORAFOR ${ }^{2}$
}

\author{
${ }^{1}$ University of Nigeria, Faculty of Veterinary Medicine, Department of Veterinary Pathology and Microbiology, Nsukka, \\ Nigeria; madubuike.anyanwu@unn.edu.ng (*correspondingauthor); ifeoma.cugwu@unn.edu.ng; martins2babe@gmail.com \\ ${ }^{2}$ University of Nigeria, Faculty of Veterinary Medicine, Department of Veterinary Medicine, Nsukka, Nigeria; obianuju.okorafor@unn.edu.ng
}

\begin{abstract}
This study was conducted to isolate generic extended-spectrum $\beta$-lactam-resistant enterobacteria from household dogs in Nigeria, and to determine the antibacterial resistance profile of the isolates. Rectal swabs were collected from 100, randomly selected, apparently healthy household dogs. Isolation of ESBL-resistant enterobacteria was done using Mac Conkey agar supplemented with $4 \mu \mathrm{g} / \mathrm{ml}$ of ceftazidime. Phenotypic characterization of the isolates to generic level was done following standard biochemical methods. Phenotypic resistance of the isolates to antibacterial agents was determined using the disc diffusion method. Out of 27 ESBL-resistant enterobacterial isolates, 40.7\% were Escherichia coli, 37\% were Klebsiella species, 18.5\% were Salmonella species, while 3.7\% was Proteus species. Resistance of the E. coli isolates was $81.8 \%$ to ampicillin, $27.2 \%$ to streptomycin, $54.5 \%$ to ciprofloxacin and tetracycline, $45.4 \%$ to enrofloxacin, $90.9 \%$ to sulphamethoxazole/trimethoprim, $9.1 \%$ to amoxicillin/clavulanic acid and $0 \%$ resistant to gentamicin. Resistance of the Klebsiella isolates was $80 \%$ to ampicillin, $20 \%$ to streptomycin and amoxicillin/clavulanic acid, 30\% to ciprofloxacin and enrofloxacin, $60 \%$ to tetracycline, $90 \%$ to sulphamethoxazole/trimethoprim and $10 \%$ to gentamicin. Resistance of the Salmonella isolates was $100 \%$ to sulphamethoxazole/trimethoprim, $80 \%$ to gentamicin and ampicillin, $60 \%$ to streptomycin and tetracycline, $20 \%$ to amoxicillin/clavulanic acid, and $0 \%$ to ciprofloxacin and enrofloxacin. The Proteus isolate was resistant to streptomycin and gentamycin. All the isolates were resistant to ceftazidime and cefotaxime. Resistance of the isolates to more than 3 classes of antibacterial agents tested was $81.8 \%$ for E. coli, $70 \%$ for Klebsiella and $100 \%$ for Salmonella, respectively. This study has shown that household dogs in Nigeria, are colonized by ESBL-resistant Enterobacteriaceae and are potential reservoirs and disseminators of these organisms.
\end{abstract}

Keywords: antibiogram, $\beta$-lactam-resistant, Enterobacteriaceae, extended-spectrum, companion animals, canine

\section{Introduction}

Antimicrobial resistance is a serious threat to the future of antimicrobial chemotherapy, and negatively impacts the health of human and animals (WHO, 2014; Morison and Rubin, 2015). The one health oriented approach in curbing antimicrobial resistance involved assessment of the impact of animals in close association with humans (Guerra et al., 2014; Schaufler et al., 2015). Companion animals such as dogs represent potential sources of spread of ESBL-resistant organisms owing to the extensive use of antimicrobials as those used in humans, especially $\beta$-lactams, in veterinary practices dealing with small animals, and their close contact with humans (Guardabassi et al., 2004; Ma et al., 2009; Ewers et al., 2012; Hordjik et al., 2013; Tamang et al., 2012). Extended- spectrum $\beta$-lactams (i.e. $3^{\text {rd }}-[$ e.g. ceftazidime, cefpodoxime, etc and $4^{\text {th }}$-generation cephalosporins) are critical drugs in medicine, used for the treatment of bacterial infections (especially multidrug-resistant infections) in humans and companion animals (WHO, 2011; Ewers et al., 2012; Hordijk et al., 2013; Tuerena et al., 2016). Inappropriate use of extended-spectrum $\beta$-lactams especially in developing nations (due to lack of strict policy regarding the use of antibacterial agents) has led to selection pressure and development of resistance to these drugs by enterobacteria (Ugwu et al., 2015). Resistance to extended-spectrum $\beta$-lactams in enterobacteria is mediated mainly by extended-spectrum $\beta$-lactamases (ESBLs) (Tamang et al., 2012; Ljungquist et al., 2016). ESBL-resistant enterobacteria are multidrug-resistant and exhibit resistance to all $\beta$-lactams and many classes of antibacterials including 
aminoglycosides, fluoroquinolones, phenicols, lincosamides, potentiated sulfonamides and tetracycline (Coque et al., 2008; Dierikx et al., 2012; Schaufler et al., 2015). Therapeutic options for treating infections associated with ESBL-resistant organisms are often limited due to phenotypic multidrug resistance (Dierikx et al., 2012; Schaufler et al., 2015). It is well known that ESBL-resistant enterobacteria are common cause of community and hospital-associated infections in humans and companion animals (Sun et al., 2010; Rubin and Pitout, 2014). These infections are often fatal due to limited therapeutic options, resorting to the use of carbapenems is usually the treatment option (Dierikx et al., 2012; Blaak et al., 2014). This has resulted in increasing numbers of isolation of carbapenem-resistant organisms (superbugs) from humans and animals, including companion animals, worldwide (Shaheen $e t$ al., 2013; Guerra et al., 2014; Yousfi et al., 2015). Determination of phenotypic antibacterial resistance profile of ESBL-resistant enterobacteria is useful for empirical treatment of infections associated with such organisms (Ugwu et al., 2015; Schaufler et al., 2015).

Direct physical contact between dogs and humans, especially dog owners/caretakers, children and veterinarians, has been identified as a putative risk for the spread of ESBLresistant enterobacteria (Weiler et al., 2011; Meyer et al., 2012; Schaufller et al., 2015; Ljungquist et al., 2016). As commensal organisms in the intestinal tract of animals, ESBL-resistant enterobacteria haboured by dogs may be discharged into the environment, thereby serving as disseminators of genes encoding $\beta$-lactam resistance (Costa et al., 2008; Pfeiffer et al., 2010; Tuerena et al., 2016). Beta-lactam resistance genes present in the discharged enterobacteria could be acquired by horizontal transfer to pathogenic human bacteria, thereby complicating infections and compromising antibacterial therapy in carriers (Caratolli et al., 2005; Ugwu et al., 2015; Ljungquist et al., 2016).

Reports on the occurrence of probable exchange of ESBLresistant organisms between humans and animals raised questions on the role of companion animals as reservoirs of these organisms (Weiler et al., 2011; Schmeidel et al., 2014). There have been calls for assessment of companion animals as potential reservoirs and disseminators of ESBL-resistant organisms (Ewers et al., 2011; Weiler et al., 2011; Cozma et al., 2015a). Since the first report on isolation of ESBL-resistant $E$. coli in faecal sample of a laboratory dog in Japan (Matsumoto $e t$ al., 1998), improvements have been made on isolation of ESBL-resistant enterobacteria from clinical samples of dogs worldwide (Feria et al., 2002; Lim et al., 2009; Gibbson et al., 2010; O'Keefe et al., 2010; Timofte et al., 2011; Shaheen et al., 2011; Huber et al., 2013; Dierikx et al., 2012; Weiler et al., 2014; Rzewuska et al., 2015). Evidences support zoonotic transmission of ESBL-resistant enterobacteria from companion animals to humans and vice versa (Weiler $e$ e al., 2011; Meyer et al., 2012; Rubin and Pitout, 2014; Carvalho et al., 2016; Ljungquist et al., 2016).

Surveillance studies to assess dogs as potential reservoirs and disseminators of ESBL-resistant bacteria have been conducted in several countries in Europe (Costa et al., 2004; Caratolli $e t$ al., 2005; Poirel et al., 2013; Gandolfi-Decristophoris et al., 2013; Hordijk et al., 2013; Johard et al., 2015; Cozma et al., 2015a, Cozma et al., 2015b; Baede et al., 2015; Ljungquist et al., 2016), South America (Moreno et al., 2008; Rocha-Gracia et al., 2015; Oleivera et al., 2016; Carvalho et al., 2016), Asia (Sun et al., 2010; So et al., 2012; Tamang et al., 2014) and Australia (Sidjabat et al., 2007). No single report regarding the presence of ESBL-resistant enterobacteria in companion animals in Nigeria, exist in the literature whereas most Nigerian households keep dogs as pets, guard and/or hunting dogs. Reports in the literature which assessed animals as reservoirs of ESBL-resistant organisms in Nigeria, were those from food animals (Chah and Oboegbulem, 2007; Eze et al., 2013; Duru et al., 2013; Inwezerua et al., 2014; Olowe et al., 2015; Ugwu et al., 2015; Torres et al., 2015). This may paint a picture that only food animals are reservoirs of ESBL-resistant organisms in Nigeria. This is a matter of concern because companion animals in Nigeria may harbour these multidrug-resistant organisms and continually serve as source of dissemination of ESBL-encoding genes to the gut microbiota of animals and humans. Therefore, there is need to screen companion animals in Nigeria as potential reservoirs of ESBL-resistant organisms. This will help in evaluation of trends, identification of mitigation strategies and empirical treatment of infections associated with these organisms (Ugwu et al., 2015). The objective of this study, therefore, was to isolate ESBL-resistant enterobacteria from household dogs in Nsukka, Southeast, Nigeria, and to determine the antibacterial resistance profile of the isolates.

\section{Materials and Methods}

\section{Sampling}

This cross-sectional study was conducted between January and July, 2016. Households with dogs in Nsukka Southeast, Nigeria, were identified purposively, using snowballing technique. Rectal swabs were collected using sterile swab sticks from 100 (one dog per household), randomly selected apparently healthy dogs of varied breeds, sexes and ages (puppies and adults). Distinguishing body marks of each dog was appropriately noted and each house was visited once to avoid the possibility of re-sampling. The samples were transported aseptically in ice packs and processed within 2 hours of collection in the Veterinary Microbiology Laboratory, Department of Veterinary Pathology and Microbiology, University of Nigeria, Nsukka.

\section{Isolation and generic identification of ESBL-resistant enterobacteria from dogs}

The swabs were cultured on Mac Conkey agar supplemented with $4 \mu \mathrm{g} / \mathrm{ml}$ of ceftazidime and incubated at $37^{\circ} \mathrm{C}$ for 24 hours aerobically. The morphology of different colonial types were described and recorded appropriately. Purification of the isolates was done by sub-culturing on plain Mac Conkey agar and incubated at $37^{\circ} \mathrm{C}$ for 24 hours. Pure cultures of the isolates were then inoculated onto nutrient agar slants, incubated at $37{ }^{\circ} \mathrm{C}$ for 24 hours and stored in refrigerator at $4{ }^{\circ} \mathrm{C}$ as stock cultures until needed for further analysis. Phenotypic characterization of the isolates to generic level was done by subjecting them to various tests such as Gram staining, catalase, urease, oxidase, indole, methyl red, triple sugar iron agar and citrate test, and sub-culturing on eosin methylene blue agar following standard methods. 
24

Determination of the antibiogram of the ESBL-resistant enterobacterial isolates from dogs

Antibacterial resistance/susceptibility of the ESBL-resistant isolates was determined by the disc diffusion method (CLSI 2012). The isolates were sub-cultured on nutrient agar, incubated at $37^{\circ} \mathrm{C}$ for 24 hours. Then, colonies of each of the isolate were adjusted to $0.5 \mathrm{McFarland}$ 's turbidity standard (equivalent to $1 \times 10^{8}$ colony forming unit $/ \mathrm{ml}$ ) in sterile nutrient broth. The standardized broth cultures were incubated for 10 minutes at $37^{\circ} \mathrm{C}$ and then inoculated onto sterile Mueller-Hinton agar using sterile swab sticks. Ten antibacterial agents ( Oxoid $^{\circ}$ ) belonging to 6 antibacterial classes were used and they included: ceftazidime $(30 \mu \mathrm{g})$, cefotaxime $(30 \mu \mathrm{g})$, ampicillin (10 $\mu \mathrm{g})$, amoxicillin/clavulanic acid (20/10 $\mu \mathrm{g})$, tetracycline $(30 \mu \mathrm{g})$, streptomycin $(10 \mu \mathrm{g})$, gentamicin $(10$ $\mu \mathrm{g})$, ciprofloxacin (5 $\mu \mathrm{g})$, enrofloxacin $(5 \mu \mathrm{g})$ and sulphamethoxazole/trimethoprim $(25 \mu \mathrm{g})$. The discs were placed strategically on the inoculated Mueller-Hinton agar plates. The plates were incubated at $37^{\circ} \mathrm{C}$ for 24 hours. After incubation, the zone of inhibition around each disc was measured with a meter rule. Each test was performed in triplicate and the mean inhibitory zone diameter (IZD) calculated to the nearest whole millimeter $(\mathrm{mm})$ for each isolate and each antibacterial agent. The IZD was interpreted as susceptible, intermediate or resistant according to the Clinical and Laboratory Standards Institute (CLSI) (2014) criteria for aerobic isolates.

\section{Results}

\section{Prevalence of generic ESBL-resistant enterobacterial isolates from dogs}

Out of the total of 100 rectal swab samples processed for isolation of ESBL-resistant enterobacteria, 27 gave positive culture. Of the 27 ESBL-resistant isolates, 11 were Escherichia coli, 10 were Klebsiella species, 5 were Salmonella species, while 1 was Proteus species (Fig. 1).

\section{Antibiogram of generic ESBL-resistant enterobacterial isolates from dogs \\ Out of the 11 ESBL-resistant E. coli isolates, all were} resistant to ceftazidime and cefotaxime, $81.8 \%$ to ampicillin, $27.2 \%$ to streptomycin, $54.5 \%$ to ciprofloxacin and tetracycline, $45.4 \%$ to enrofloxacin, $90.9 \%$ to sulphamethoxazole / trimethoprim, and $9.1 \%$ to amoxicillin / clavulanic acid (Fig. 2). None of the E. coli isolates was resistant to gentamicin.

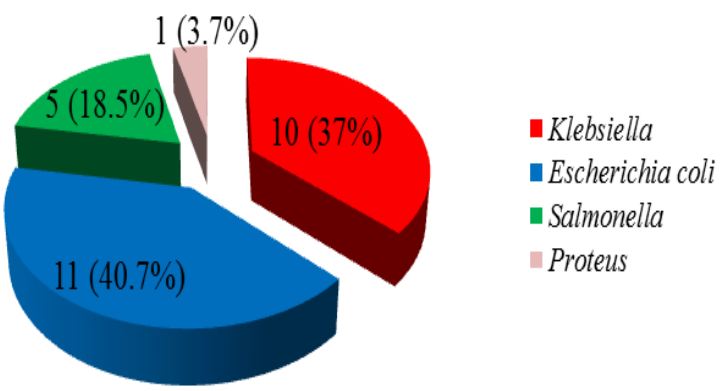

Fig. 1. Prevalence of extended-spectrum $\beta$-lactam-resistant enterobacterial isolates from dogs
Out of the 10 ESBL-resistant Klebsiella isolates, all (100\%) were resistant to ceftazidime and cefotaxime, $80 \%$ to ampicillin, $20 \%$ to streptomycin and amoxicillin/clavulanic acid, $30 \%$ to ciprofloxacin and enrofloxacin, $60 \%$ to tetracycline, $90 \%$ to sulphamethoxazole / trimethoprim and $10 \%$ to gentamicin (Fig. 3).

Out of the 5 ESBL-resistant Salmonella isolates, all (100\%) were resistant to ceftazidime, cefotaxime and sulphamethoxazole / trimethoprim, $80 \%$ to gentamicin and ampicillin, $60 \%$ to streptomycin and tetracycline, and $20 \%$ to amoxicillin / clavulanic acid (Fig. 4). None of the isolates was resistant to ciprofloxacin and enrofloxacin.

The ESBL-resistant Proteus isolate was resistant to ceftazidime, cefotaxime, streptomycin and gentamicin.

Out of the 11 E. coli isolates, 1 was resistant to one and two classes of the antibacterial agents tested while 9 were resistant to 3 or more classes (Fig. 5). Of the 10 Klebsiella isolates, 1 was resistant to one class of antibacterial agents tested, 2 were resistant to two classes while 7 were resistant to 3 or more antibacterial classes tested. All the Salmonella isolates were resistant to 3 or more classes of the antibacterial agents tested. The Proteus isolate exhibited resistance to two classes of antibacterial agents tested.

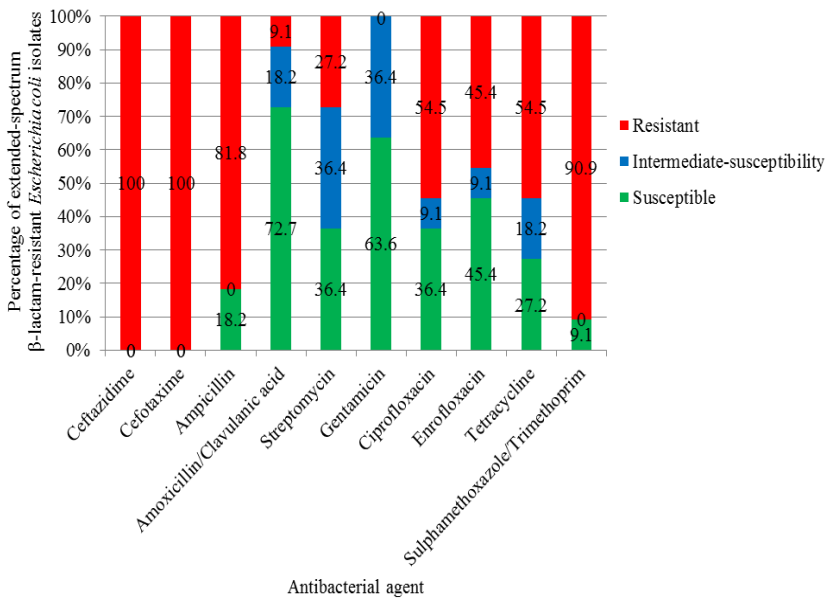

Fig. 2. Antibiogram of 11 generic extended-spectrum $\beta$-lactamresistant Escherichia coli from dogs

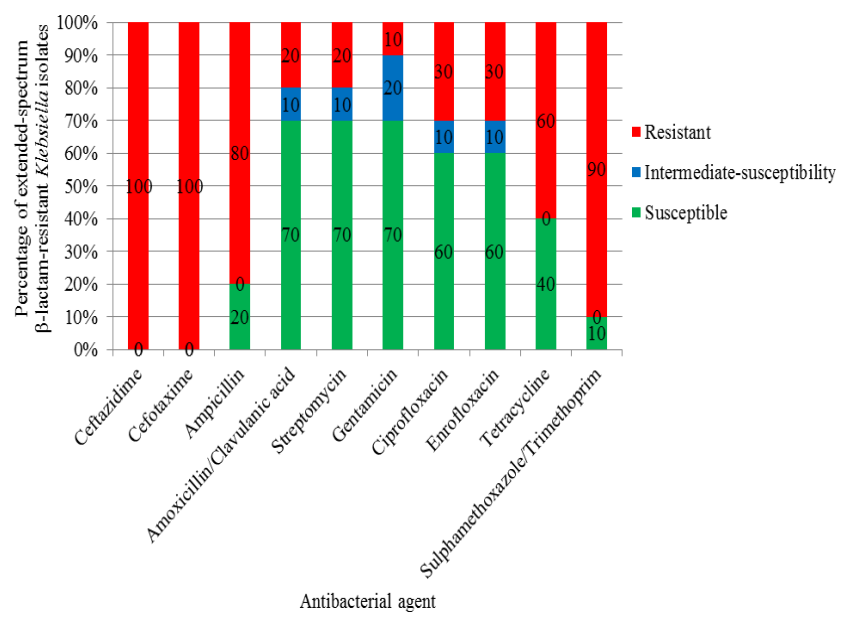

Fig. 3. Antibiogram of 10 generic extended-spectrum $\beta$-lactamresistant Klebsiella isolates from dogs 


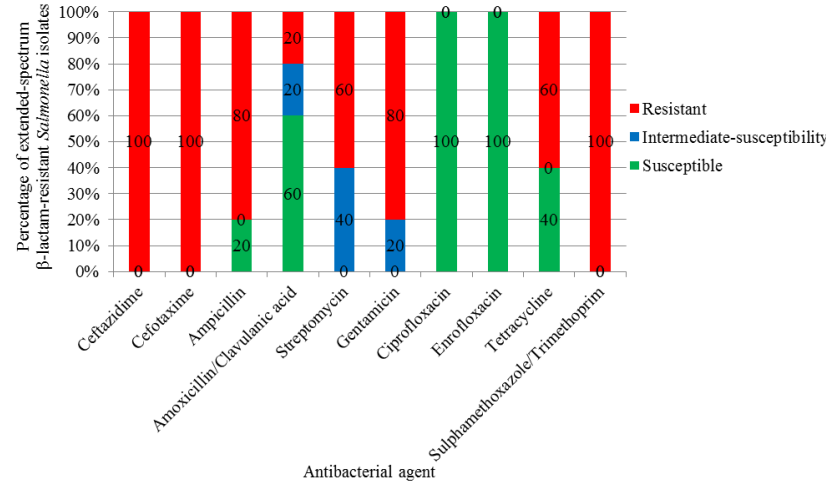

Fig. 4. Antibiogram of 5 generic extended-spectrum $\beta$-lactamresistant Salmonella isolates from dogs

\section{Discussion}

Isolation of ESBL-resistant enterobacteria from 27 dogs (27\%) among 100 apparently healthy dogs in this study, suggested a high prevalence of ESBL-resistant enterobacteria colonization of household dogs in Nsukka. This finding calls for concern because these dogs potentially serve as reservoir and disseminators (by faecal shedding) of these organisms into the environment thereby posing health threat to individuals that get in contact with them (Moreno et al., 2008; Sun et al., 2010; Meyer et al., 2012; Belas et al., 2014; Torkan et al., 2015; Carvalho et al., 2016). Environmental contamination by the isolates could result in their entry into the food chain (Woerther et al., 2013; Belas et al., 2014; Schaufler et al., 2015), thereby magnifying the health threat posed to humans and animals. Dog owners, children and veterinarians, could acquire these organisms following contact with faeces from the dogs or formites contaminated by the organisms (Sun et al., 2010; Ewers et al., 2012; Belas et al., 2014; Ljungquist et al., 2016; Torkan et al., 2016). Moreover, contact with companion (pet) animals have been linked with colonization of intercontinental travelers by ESBL-resistant enterobacteria; therefore, these organisms could spread from Nigeria to other parts of the globe (Ewers et al., 2010; van der Bij and Pitout, 2012; Belas et al., 2014; Woerther et al., 2013; Ljungquist et al., 2016).

The $27 \%$ ESBL-resistant enterobacteria isolation prevalence recorded in this study, is higher than 17 and $12 \%$ ESBL-producing enterobacteria isolation prevalence in rectal swabs of 174 and 202 healthy dogs/cats reported in Switzerland, respectively (Gandolfi-Decristophoris et al., 2013). It is also higher than 9, 28.9, 2 and 3.6\% ESBLresistant/producing enterobacterial isolation prevalence in 22 household dogs in Sweden, 58 faecal samples of healthy dogs, among clinical enterobacterial isolates from 65 dogs/cats/horses and $608 \mathrm{dogs} /$ cats reported by Ljungquist et al. (2016), Cozma et al. (2015a), Dierikx et al. (2012) and Bogaerts et al. (2015) in Sweden, Romania, The Netherlands and Europe, respectively. The result is however lower than 62.1 and 30\% ESBL-producing enterobacterial isolation prevalence in faecal samples of 29 healthy dogs/cats and among $110 \mathrm{dogs} / \mathrm{cats} /$ others reported by Cozma et al. (2015b) and Poirel et al. (2013) in Romania and France, respectively. A longitudinal study conducted in the Netherlands, reported 45$63 \%$ ESBL-producing enterobacterial isolation prevalence in

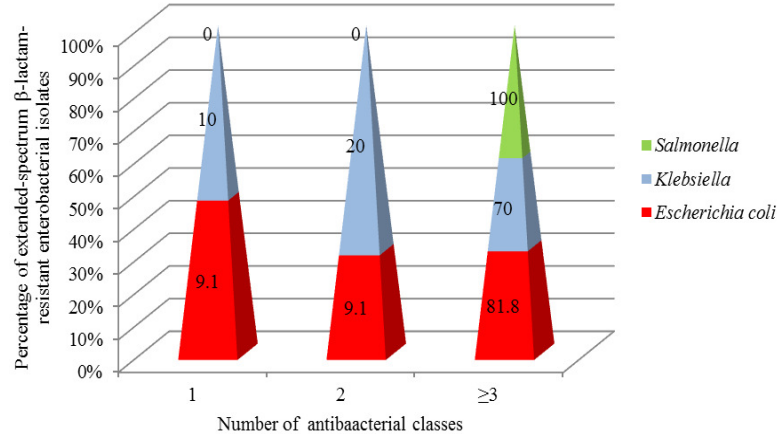

Fig. 5. Resistance of extended-spectrum $\beta$-lactam-resistant enterobacterial isolates to antibacterial classes

faecal samples of 38 dogs (Baede et al., 2015); this result is also higher than the ESBL-resistant enterobacteria prevalence $(27 \%)$ recorded in the present study. The variation in enterobacteria isolation prevalence reported in these studies may be due to disparity in the method of isolation/processing, media/concentration of extended-spectrum cephalosporin used for the isolation and type of sample (whether clinical or non-clinical) used in the various studies, and usage of extendedspectrum $\beta$-lactams in the various study areas (Damborg et al., 2015). ESBL-resistant Enterobacteriaceae have been isolated elsewhere using the same concentration of extended-spectrum cephalosporin as in this study (Schaufler et al., 2015)

Isolation of four enterobacteria genera (E. coli, Klebsiella, Salmonella and Proteus) in this study, suggested acquisition / transfer of genes encoding ESBLs by/among the enterobacterial isolates within the gut of the sampled dogs. Possible sources of isolates in this study include contaminated food (including pet foods and meat/meat products) and drinking water, dogs' environment (walking and living environment), veterinary hospital environment on previous visitation, owners/handlers, and/or formites contaminated by the ESBL-resistant organisms which the dogs had contact with during straying/scavenging (Caratolli et al., 2005; Murphy et al., 2010; Schaufler et al., 2015; Nilsson, 2015; Tuerena et al., 2016; Ljungquist et al., 2016). It is also possible that the isolates acquired genes encoding ESBLs from other organisms (commensals or transient pathogens) in the sampled dogs following previous exposure to extended-spectrum $\beta$-lactam used in treating the animals (Caratolli et al., 2005; Teurena et al., 2016; Ljungquist et al., 2016).

In this study, $40.7 \%$ E. coli isolation prevalence compared against Klebsiella (37\%), Salmonella (18.5\%) and Proteus (3.7\%), suggested that E. coli may be the dominant ESBLresistant enterobacteria genus colonizing dogs in Nsukka. The result also suggested that the $E$. coli isolates may have acquired ESBLs-encoding genes more than the other genera. It has been reported that $E$. coli acquires ESBLs more than any other enterobacterial genera in companion animals (SVARM, 2011). The highest $E$. coli isolation prevalence $(40.7 \%)$ recorded in this study differs from the result of a similar study conducted in Enugu State, Nigeria, in which Klebsiella was the dominant (67.4\% isolation prevalence) enterobacteria genus isolated from healthy pigs (Ugwu et al., 2015). The result of this study, also contrasted Poirel et al. (2013) who reported Klebsiella, with 
26

51.5\% prevalence, as the dominant genus among 33 ESBLproducing enterobacterial isolates from dogs/cats/others in France.

The $40.7 \%$ E. coli isolation prevalence in this study is higher than 17, 3.2, 24.5, 20, 1.9 and 3\% ESBL-resistant/producing $E$. coli isolation prevalence reported in faecal samples of 53 healthy dogs in Mexico (Rocha-Gracia et al., 2015), oral swabs of 31 healthy dogs in Brazil (Oliveira et al., 2016), faecal samples of 109 healthy dogs in China (Sun et al., 2010), faecal/urine samples of companion animals in Chile (Moreno et al., 2008), 209 dog faecal deposits in Denmark (Damborg et al., 2015) and among 944 clinical $E$. coli isolates from companion animals in America (Shaheen et al., 2011), respectively. It is also higher than 16, 7, 6.6 and 2.6\% ESBL-resistant/producing E. coli isolation prevalence in 100 extra-clinical faecal samples of dogs in Germany (Schaufler et al., 2015), among 445 clinical E. coli isolates from companion animals in United Kingdom (UK) (Timofte $e t$ al., 2016), faecal samples of 75 healthy dogs/cats (Costa et al., 2004) and 39 healthy dogs (Costa et al., 2008) reported in Portugal, respectively. Pomba et al. (2009), Tamang et al. (2012), Rzewuska et al. (2015), Caratolli et al. (2005), O'Keefe et al. (2010), Huber et al. (2013) and Feira et al. (2002) reported 1.6, 2.9, 28.2, 7, 5, 7.5 and 1.4\% ESBLproducing/resistant $E$. coli isolation prevalence among 61, 730, 628, 298, 257, 107 and 72 E. coli isolates from urine/urinary tract of sick dogs in Portugal, dogs/cats in Poland, intestinal $E$. coli isolates from stray dogs in South Korea, sick/healthy $\mathrm{dogs} / \mathrm{cats} / \mathrm{rat}$ in Italy, urine/urinary tract of sick dogs/cats in America and Switzerland, and healthy dogs in Portugal, respectively. These results are also lower than $40.7 \%$ E. coli isolation prevalence recorded in the current study. In Brazil, Carvalho et al. (2016) reported 28.5\% ESBL-resistant / producing $E$. coli isolation prevalence among 42 faecal $E$. coli isolates from household dogs, a finding that is also lower than the result (40\%) of the current study. An Iranian study also reported lower ESBL-producing $E$. coli isolation prevalence in faecal samples of healthy cats (Akhtardanesh et al., 2015). The $E$. coli isolation prevalence in the present study, is however, lower than ESBL-producing $E$. coli isolation prevalence recorded among 20 faecal ESBL-producing enterobacterial isolates from healthy dogs/cats in Romania (Cozma et al., 2015b), 63 faecal E. coli isolates from hospitalized dogs in South Korea (So et al., 2012), 33 ESBL-producing enterobacterial isolates from companion animals in France (Poirel et al., 2013), 15 E. coli isolates from urine samples of 138 sick cats in Italy (Nebia et al., 2014) and in faecal samples of 20 each of healthy and diarrhoeic dogs in the Netherlands (Hordjik et al., 2013), respectively. It is also lower than 62\% ESBL-resistant $E$. coli isolation prevalence in faecal samples from 13 sick dogs reported by Damborg et al. (2011) in Denmark. Murphy et al. (2009) reported 0\% ESBL-resistant $E$. coli isolation prevalence among 188 dogs in America, a finding that contrasted the result of the present study.

The $37 \%$ Klebsiella isolation prevalence in this study is higher than 15\% ESBL-producing Klebsiella isolation prevalence among 20 faecal ESBL-producing enterobacterial isolates from healthy dogs/cats reported by Cozma $e t$ al. (2015b) in Romania. The least isolation prevalence of Proteus
(3.7\%) in this study may be attributed to the fact that this organism is mostly isolated from urinary tract of infected dogs than faecal samples of healthy animals (Feria et al., 2012). The variation in isolation prevalence of ESBL-resistant enterobacteria genera reported in these studies, may be related to the differences in sample size, type of sample processed (whether clinical or non-clinical), rate of contamination of dog's food/drinking water or environment by ESBL-resistant enterobacteria and usage of extended-spectrum cephalosporins in the various study areas (Hordjik et al., 2013; Baede et al., 2015).

It is recommended that phenotypic ESBLs production be detected using extended-spectrum $\beta$-lactam (such as ceftazidime, cefotaxime or cefpodoxime) with/without clavulanate in double disc synergy test (DDST) (CLSI, 2012). Recently, it was recommended that when using the new interpretative breakpoints (for EUCAST and CLSI), routine ESBL testing is no longer necessary and reporting of susceptibility results to penicillins and cephalosporins for ESBLs-producing Enterobacteriaceae should be 'as found' (Lerclerq et al., 2013; Timofte et al., 2016; CLSI, 2014). In the hereby experiment, resistance of the isolates to the drugs were tested using single discs. The fact that all the isolates (100\%) in this study exhibited resistance to extended-spectrum $\beta$-lactams (ceftazidime and cefotaxime) tested, further suggested they produced ESBLs. The high rate of extended-spectrum $\beta$-lactam resistance in this study may be as a result of frequent use of extended-spectrum $\beta$-lactams in small animal medicine in Nigeria. Moreover, extended-spectrum $\beta$-lactams are also frequently used in Nigerian hospitals, and high prevalence of ESBL-resistant organisms in food animals, is a good indication that the drugs are used in these animals in the country (Torres et al., 2015; Ugwu et al., 2015). Thus, many potential sources of ESBLs genes existed, acquisition of the genes by the isolates might have been facilitated by poor personal and public hygiene in Nigeria (Woerther et al., 2013; Schaufler et al., 2013). The high extended-spectrum $\beta$-lactam resistance in this study, calls for real concern, because, if the selective pressure resulting from indiscriminate use of these critical drugs is not curtailed (by implementing antibacterial stewardship programs), the use of carbapenems would be resorted for treating multidrug-resistant infections in companion animals in Nigeria. Consequently, this would result in spread of superbugs (associated with untreatable and highly fatal infections) in companion animals and humans in Nigeria. The $100 \%$ cefotaxime resistance in this study is similar to $100 \%$ cefotaxime resistance reported among 32 clinical ESBLresistant $E$. coli isolates from companion animals in UK (Timofte et al., 2016); 4 faceal ESBL-producing E. coli isolates from healthy dogs in Portugal (Costa et al., 2004), 8 uropathogenic ESBL-producing $E$. coli isolates from dogs/cats in Switzerland (Huber et al., 2013) and 21 ESBL-resistant $E$. coli isolates from sick/healthy pets in Italy (Caratolli $e t$ al., 2005), respectively. The $100 \%$ ceftazidime resistance in this study is also similar to $100 \%$ ceftazidime resistance reported by Timofte et al. (2016). Tuerena et al. (2016) reported 27.2\% extended-spectrum cephalosporins resistance among $E$. coli isolates from companion animals/environment/hospital in Liverpool, United Kingdom. This finding is lower than the extended-spectrum cephalosporins resistance recorded in this experiment. 
High ampicillin resistance observed in the hereby experiment, further suggested production of ESBLs by the isolates. Ampicillin is a $\beta$-lactam which is rendered ineffective by ESBLs (Ugwu et al., 2015). The high ampicillin resistance in this study could also be as a result of production of $\beta$-lactamases (a major mechanism of $\beta$-lactam resistance) which hydrolysed the $\beta$-lactam ring of the drug to penicilloic acid, change in cell wall permeability, expression of active efflux pump, gene mutation and/or altered penicillin binding protein (PBP) receptors in the isolates (Urmunova, 2015). The rate of ampicillin resistance (80-81.8\%) among isolates in this study is lower than that (100\%) observed among isolates of the same genera in healthy pigs in Enugu State, Nigeria (Ugwu et al., 2015).

The $81.8 \%$ E. coli ampicillin resistance in this study is lower than $100 \%$ ampicillin resistance among 21 ESBL-resistant $E$. coli isolates from sick/healthy pets, 8 uropathogenic ESBLproducing $E$. coli isolates from dogs/cats, 32 ESBL-resistant clinical $E$. coli isolates, and 14 ESBL-resistant $E$. coli isolates from extra-clinical faecal samples of dogs reported by Caratolli et al. (2005), Huber et al. (2013), Timofte et al. (2016) and Schaufler et al. (2015) in Italy, Switzerland, UK and Germany, respectively. The variation in ampicillin resistance recorded in these studies could be related to differences in usage of ampicillin in the various study areas (Ugwu et al., 2015). Ampicillin is not known to be a drug of choice in small animal medicine in Nigeria, but it is frequently used in humans (alone or in combination with cloxacillin) and food-producing animals (Chah and Nweze, 2001; Ugwu et al., 2015). Thus, these factors could also have contributed to high ampicillin resistance observed in this study (Ljungquist et al., 2016).

The Proteus isolate in this study was susceptible to ampicillin, suggesting lack of selection against the drug. This finding suggested that, although the isolate exhibited phenotypic extended-spectrum $\beta$-lactam resistance, it may not harbour genes encoding ESBLs (Caratolli et al., 2005; Beceiro et al., 2011). It also suggested that the extended-spectrum $\beta$ lactam resistance exhibited by the Proteus isolate occurred by other mechanisms such as expression of efflux pump, plasmidmediated Amp C $\beta$-lactamases or plasmid-mediated metalo- $\beta$ lactamases (Batchelor et al., 2005; Damborg et al., 2011; Harada et al., 2014; Urmunova, 2015).

Demonstrable amoxicillin/clavulanic acid resistance among E. coli (9.1\%), Klebsiella and Salmonella (20\%) isolates in this study, suggested selection against the drug. This also suggested that Klebsiella and Salmonella isolates exerted more selection to the drug than the E. coli isolates. However, variation in number of isolates in each genus tested against amoxicillin/clavulanic acid in this study might be responsible for the discrepancies observed in the resistance rates. Nevertheless, the result suggested production of other $\beta$-lactamases such as AmpC cephalosporinases (Ben Sallem et al., 2012; Tamang et al., 2012; Timofte et al., 2016). Amp C cephalosporinases mediate resistance to $3^{\text {rd }}$-generation $\beta$-lactams/cephalosporins and are not inhibited by clavulanic acid and other $\beta$-lactamaseinhibitors such as sulbactam, avibactam and tazobactam (Ben Sallem et al., 2012; Tamang et al., 2012; Tuerena et al., 2016) while ESBLs are inhibited by clavulanic acid and other $\beta$ lactamase-inhibitors (Ben Sallem et al., 2012). Nonetheless, amoxicillin / clavulanic acid resistance observed in this experiment may be as a result of selection following the use of the drug in the animals (Seiffert et al., 2013; Tuerena $e t$ al., 2016). Potentiated amoxicillin is widely used for treating bacterial infections associated with $\beta$-lactamases in humans and small animals worldwide, including in Nigeria (Radford $e t a l$, 2011; Tuerena et al., 2016). Other possible mechanisms by which the isolates exerted selection to amoxicillin/clavulanic acid in this study include hyper-production of class A $\beta$ lactamases (TEM-1 or SHV-1), production of class D plasmidmediated enzyme, chromosomal or plasmidic class $C$ lactamase, ampicillinase $\mathrm{C}$ and/or modification of outer membrane permeability (Dwarz and Bonomo, 2010). The finding of moderate to high amoxicillin/clavulanic acid resistance in this study is a cause for some concern because the use of potentiated amoxicillin has been related to the development of ESBL resistance (especially AmpC-mediated) in enterobacterial isolates from humans and companion animals (Aldeyab et al., 2012; Johard et al., 2015; Tuerena et al., 2016). Again, organisms exhibiting resistance to potentiated amoxicillin are known to be multidrug resistant, thus posing huge health threat to the dogs and individuals (humans and animals) in contact with them (Tamang et al., 2012). However, the ESBLs produced by the isolates might also have mediated resistance to the amoxicillin component of the drug (Tuerena et al., 2016). Notably, isolates from healthy pigs in Enugu State, Nigeria, belonging to the genera reported in the present study, showed $100 \%$ amoxicillin/clavulanic acid resistance (Ugwu et al., 2015). Therefore, it is also possible that potentiated amoxicillin resistance observed in this study emanated from the food chain. The Proteus isolate in this study, was susceptible to amoxicillin/clavulanic acid, this suggested absence of selection against the drug.

The $9.1 \%$ E. coli amoxicillin/clavulanic acid resistance in this study, is higher when compared with 4 and $0 \%$ amoxicillin/clavulanic acid resistance among ESBLresistant/producing E. coli isolates from dog faecal deposits and healthy/sick dogs/cats/rat reported by Caratolli et al. (2005) and Damborg et al. (2015) in Italy and Denmark, respectively. But it is lower than 57.1 and $71.4 \%$ amoxicillin/clavulanic acid resistance among ESBL-resistant $E$. coli isolates from companion animals reported by Caratolli et al. (2005) and Schaufler et al. (2015) in Italy and Germany respectively. It is also lower than $100 \%$ amoxicillin/clavulanic acid resistance among ESBL-resistant $E$. coli isolates from companion animals in Switzerland (Huber et al., 2013) and UK (Timofte et al., 2016).

In this study, higher rate of ciprofloxacin (54.5\%) and enrofloxacin (45.4\%) resistance among the $E$. coli isolates as against the Klebsiella and Salmonella isolates with 30 and 0\% resistance to both drugs, respectively, suggested higher selection to these fluoroquinolones by the $E$. coli isolates than the other genera. The result also suggested that the $E$. coli isolates exerted selection against ciprofloxacin more than enrofloxacin. This moderate to high fluoroquinolones resistance may be due to acquisition of genes encoding fluoroquinolones resistance following use-selection pressure. This finding of moderate to high fluoroquinolones resistance in this study, calls for real concern, because these drugs are not known to be commonly used in small animal medicine in Nigeria. However, these drugs are frequently used in humans and food-producing animals in 
28

Nigeria (Ugwu et al., 2015); thus, it is possible the fluoroquinolone resistance observed in this study, emanated from the food chain and/or environment (Schaufler et al., 2015; Ljungquist et al., 2016; Tuerena et al., 2016). Moreover, because dogs are allowed to stray and scavenge in Nigeria, the sampled dogs could also have acquired bacteria which haboured fluoroquinolone determinants (served as source of transfer of these genes to the isolates) from various sources. However, the ESBLs produced by the isolates could also have mediated resistance to fluoroquinolones in the isolates (Coque et al., 2008; Huber et al., 2013). Cross resistance of ESBLresistant Enterobacteriaceae to fluoroquinolones have been reported (Jacoby, 2005; Frank et al., 2011; Schaufler et al., 2015).

Enterobacterial isolates in the same genera, obtained from healthy pigs in Enugu State, showed 12.9-50\% ciprofloxacin resistance (Ugwu et al., 2015). The $54.5 \%$ E. coli ciprofloxacin resistance in this study is higher when compared with 26,50 and $0 \%$ ciprofloxacin resistance reported by Pomba et al. (2009), Tamang et al. (2014) and Poirel et al. (2013) among 61 clinical E. coli isolates from dogs/cats in Portugal, 6 and 16 ESBL-producing E. coli isolates from stray dogs in South Korea and companion/domestic animals in France, respectively. But it is lower when compared with $100 \%$ ciprofloxacin resistance reported by So et al. (2012) and Huber et al. (2013) among 24 faecal ESBL-resistant $E$. coli isolates from hospitalized dogs in South Korea and 8 uropathogenic ESBL-producing E. coli isolates from dogs/cats in Switzerland, respectively. Timofte $e t$ al. (2016) recorded $65 \%$ ciprofloxacin resistance among clinical ESBL-resistant $E$. coli isolates from dogs in UK; this result is also higher than the findings (54.5\%) of the present study. The $45.4 \%$ E. coli enrofloxacin resistance in this study is higher than 35.7 and 20\% enrofloxacin resistance among ESBLresistant/producing $E$. coli isolates from extra-clinical faecal samples of dogs and dog faecal deposists reported by Schaufler et al. (2015) and Damborg et al. (2015) in Germany and Denmark, respectively. It is lower than 92 and 100\% enrofloxacin resistance among ESBL-producing $E$. coli isolates reported by Shaheen $e t$ al. (2011) and Huber $e t$ al. (2013) in America and Switzerland, respectively. The Proteus isolate in this study was susceptible to the fluoroquinolones (ciprofloxacin and enrofloxacin) tested.

In this study, resistance to aminoglycosides was observed among isolates in all the four genera. The rate of aminoglycoside resistance was higher among the Salmonella isolates ( $80 \%$ and $60 \%$ gentamicin and streptomycin resistance, respectively) than the E. coli and Klebsiella isolates with lower resistance to the drugs. This finding suggested that the Salmonella isolates exerted more selection to the aminoglycosides than the other genera. Salmonella have been reported to be highly resistant to aminoglycosides (CLSI, 2012; CLSI 2014). In fact, the CLSI recommended that Salmonella isolate which exhibited susceptibility to aminoglycoside, should be reported as resistant because these drugs may not be clinically effective against the isolate (CLSI, 2014). The Proteus isolate exhibited resistance to both gentamicin and streptomycin. The aminoglycosides resistance noted in this study, may be as a result of acquisition of genes encoding aminoglycoside resistance following use-selection pressure (Ugwu et al., 2015). Gentamicin and streptomycin (often combined with penicillin to exert a broad-spectrum effect) are frequently used for treating bacterial infections in humans and animals (especially in small animals) in Nigeria. It is also possible that the isolates acquired an aminoglycoside gene, which encoded resistance to the drugs (gentamicin and streptomycin) tested in this study. Febler et al. (2011) reported that a gene can encode resistance for many aminoglycosides. However, the ESBLs produced by the isolates could also have mediated the aminoglycoside resistance (Coque et al., 2008; Huber et al., 2013). Morosini et al. (2006) reported crossresistance of ESBL-resistant enterobacteria to aminoglycosides. The aminoglycoside resistance in this study calls for serious concern, because there is a high correlation between gentamicin resistance and multiresistance profiles in nosocomial strains including ESBL-resistant enterobacteria (Meirelles-Pereira $e t$ al., 2002; Carvalho et al., 2016).

The $0 \%$ E. coli gentamicin resistance in this study is similar to $0 \%$ gentamicin resistance among 8 faecal ESBL-resistant $E$. coli isolates from dogs reported by Damborg et al. (2011) in Denmark. It is lower than 64.3, 66.7, 33.3, 100 and 20\% gentamicin resistance observed among ESBL-resistant / producing E. coli isolates from extra-clinical faecal samples from dogs in Germany (Schaufler et al., 2015), stray dogs in South Korea (Tamang et al., 2012), sick/healthy dogs/cats/rat in Italy (Caratolli et al., 2005), and sick cats/dogs in Switzerland (Huber et al., 2013), respectively. The 10\% gentamicin resistance among Klebsiella isolates in this study is lower when compared with $100 \%$ gentamicin resistance among 16 ESBLproducing Klesbsiella isolates from rectal/urine samples of companion/domestic animals reported by Poirel $e t$ al. (2013) in France. The $27.2 \%$ E. coli streptomycin resistance in this study is lower than $50,66.7$ and $75 \%$ streptomycin resistance reported by Tamang et al. (2012), Caratolli et al. (2005) and Costa et al. (2004) in South Korea, Italy and Portugal, respectively. The variation in aminoglycosides resistance observed in these studies may be due to differences in their usage in the study areas and/or the number of isolates tested in the various studies. The variations may also be related to differences in the rate of production of ESBLs by the isolates as well as the rate of acquisition of aminoglycoside determinants (Coque et al., 2008; Huber et al., 2013). ESBLs have been reported to mediate resistance to aminoglycosides (Huber et al., 2013).

High $(54.5 \%$ for E. coli and $60 \%$ for Klebsiella and Salmonella) tetracycline resistance observed among the isolates in this study, suggested selection against the drug. The tetracycline resistance may be due to acquisition of genes encoding tetracycline resistance as a result of use-selection pressure. In Nigeria, 5\% (short-acting) tetracycline is frequently used (due to its broad-spectrum effect) in small animal clinical practice while the long-acting (20\%) tetracycline and other forms of the drug, are extensively used in humans and foodproducing animals (Ugwu et al., 2015). Thus, selective pressure for tetracycline resistance might have emanated from various sources. ESBLs have also been reported to mediate tetracycline resistance in enterobacteria (Huber et al., 2013). The $54 \% E$. coli tetracycline resistance in this study is higher than $12.5 \%$ tetracycline resistance among 8 ESBL-resistant $E$. coli isolates from dogs reported by (Damborg et al., 2011) in Denmark. But, it is lower than $75,95.2,75$ and $100 \%$ tetracycline 
resistance observed among ESBL-producing $E$. coli isolates from companion animals in South Korea (Tamang et al., 2012), Italy (Caratolli et al., 2005), and Portugal (Costa et al., 2004). In Switzerland (Huber et al., 2013) and UK (Timofte $e t$ al., 2016), 100\% tetracycline resistance was observed among ESBL-resistant/producing $E$. coli isolates from companion animals; this result is also higher than that of the current work. The 60\% Klebsiella tetracycline resistance observed in this study is lower when compared with $100 \%$ tetracycline resistance among ESBL-producing Klesbsiella isolates from rectal/urine samples of companion/domestic animals reported in France (Poirel et al., 2013).

In this study, tetracycline resistance was not observed in the Proteus isolate; thus, suggesting it did not exert selection against the drug. This finding suggested that the isolate may not be $P$. mirabilis which is intrinsically resistant to tetracycline (Magiorakos et al., 2011; Dierikx et al., 2012)

The 100\% sulphamethoxazole/trimethoprim resistance among Salmonella isolates in this study, suggested complete selection to potentiated sulphonamide in this genus. It also suggested that Salmonella isolates exerted selection to the drug more than the E. coli and Klebsiella isolates with 90.9 and $90 \%$ sulphamethoxazole/trimethoprim resistance, respectively. The high potentiated sulphonamide resistance among the isolates may be due to selection following acquisition of genes encoding potentiated sulphonamide resistance (Torkan et al., 2015). Sulphamethoxazole/trimethoprim is frequently used in humans and animals (mostly in canine clinical practice) in Nigeria (Ugwu et al., 2015). The $90.9 \%$ E. coli sulphamethoxazole/trimethoprim resistance in this study is higher than 59, 60 and 58.3\% sulphamethoxazole / trimethoprim resistance reported by Timofte et al. (2016), Damborg et al. (2015) and Tamang et al. (2012) in UK, Denmark and South Korea, respectively. It is lower than 95.2, 100 and $85.7 \%$ sulphamethoxazole trimethoprim resistance among ESBL-resistant/producing E. coli isolates from companion animals, and extra-clinical faecal samples of dogs reported by Caratolli et al. (2005), Huber et al. (2013) and Schaufler et al. (2015) in Italy, Switzerland and Germany, respectively. The $90 \%$ sulphamethoxazole / trimethoprim among Klebsiella isolates in this study is lower when compared with $100 \%$ sulphamethoxazole/triemthoprim resistance among 16 ESBL-producing Klesbsiella isolates from faecal/urine samples of companion/domestic animals reported by Poirel et al. (2013) in France. Variations in potentiated sulphonamide resistance in these studies may be related to differences in method used for antimicrobial sensitivity and drug concentration in the various studies, and usage of potentiated sulphonamide in the various study areas. In the present study, selection against potentiated sulphonamide was not observed in Proteus isolate.

Resistance to three or more classes of antibacterial agent implies multidrug resistance (Tenover 2006; Nam et al., 2010; Rzewuska et al., 2015). In this study, resistance of all the Salmonella isolates to three or more of the antibacterial agents suggested that isolates in the genus exhibited higher multidrug resistance when compared with the multidrug resistance of the E. coli (81.8\%) and Klebsiella (70\%) isolates. The $81.8 \%$ E. coli multidrug resistance in this study is lower than 93.9 and $100 \%$ multidrug resistance among ESBL-resistant/producing $E$. coli isolates from extra-clinical faecal samples of dogs and healthy pets reported by Schaufler et al. (2015) and Caratolli et al. (2005) in Germany and Italy, respectively. The result is however higher than 31.3 and $66.7 \%$ multidrug resistance among 112 ESBL-resistant $E$. coli isolates from companion/domestic animals and 12 ESBL-producing E. coli isolates from stray dogs reported by Filipovic et al. (2007) and Tamang et al. (2012) in Montenegro and South Korea, respectively. The Proteus isolate in this study exhibited resistance to two classes (B-lactam and aminoglycoside) of antibacterial agents tested. Therefore, it may not be regarded as a multidrug-resistant strain. The high multidrug resistance exhibited among enterobacterial isolates in this study, poses huge health threat to individuals (humans and animals) that have direct physical (dog owners, dog caretakers/handlers, children and veterinarians) and/or indirect (general public via contact with the primary contacts, environment and the food chain) contact with the dogs (Torkan et al., 2015; Srisanga et al., 2016). These individuals could acquire these multidrug resistant organisms. Consequently, there could be compromise in antibacterial therapy in colonized and/or infected individuals (Ugwu et al., 2015).

\section{Conclusions}

This study has shown that ESBL-resistant enterobacteria are harboured by a sizeable percentage $(27 \%)$ of household dogs in Nsukka, Southeastern Nigeria. E. coli is the most prevalent genus of ESBL-resistant enterobacteria colonizing household dogs within the study area. Thus, household dogs in the study area are potential reservoirs and disseminators of ESBL-resistant enterobacteria and genes encoding ESBLs production. This has tremendous impact on the ecology of antibacterial resistance and potentially it can impact negatively on the food chain. Therefore, attention should be paid on the use of extended-spectrum $\beta$-lactams in companion animals in Nigeria. However, further studies to determine the types of ESBLs genes harboured by the isolates are recommended.

\section{References}

Akhtardanesh B, Ghanbarpour R, Yazdani E (2015). Determination of extended-spectrum beta-lactamases genes and antibiotic resistance patterns in Escherichia coli isolates from healthy cats. Journal of Medical Bacteriology 4(5-6):1-6.

Aldeyab MA, Harbarth S, Vernaz N, Kearney MP, Scott MG,Darwish Elhajij FW, Aldiab MA, McElnay JC (2012). The impact of antibiotic use on the incidence and resistance pattern of extended-spectrum betalactamase producing bacteria in primary and secondary healthcare settings. British Journal ofClinical Pharmacology 74:171-179.

Baede VO, Wagenaar JA, Broens EM, Duim B, Dohmen W, Nijsse R, Timmerman AJ, Hordijk J (2015). Longitudinal study of extendedspectrum--3lactamase- and AmpC-producing Enterobacteriaceae in household dogs. Antimicrobial Agents and Chemotherapy 59:31173124.

Batchelor M, Threlfall EJ, Liebana E (2005). Cephalosporin resistance among animal-associated enterobacteria: a current perspective. Expert Review of Anti-infective Therapy 3:403-417. 
30

Beceiro A, Maharjan S, Gaulton T, Doumith M, Soares NC, Dhanji H, Warner M, Doyle M, Hickey M, Downie G, Bou G, Livermore DM, Woodford N (2011). False extended-spectrum \{beta\}-lactamase phenotype in clinical isolates of Escherichia coli associated with increased expression of OXA-1 or TEM-1 penicillinases and loss of porins. Journal of Antimicrobial Chemotherapy 66:2006-2010.

Ben Sallem R, Ben Slama K, Saenz Y, Rojo-Bezares B, Estepa V, Jouini A, Gharsa H, Klibi N, Boudaous A, Torres C (2012). Prevalence and characterization of extended-spectrum beta-lactamase (ESBL)-and CMY-2-producing Escherichia coli isolates from healthy food producing animals in Tunisia. Foodborne Pathogens and Disease 9(12):11371142.

Blaak H, Hamidjaja RA, van Hoek AHAM, de Heer L, Husman AM, Schets FM (2014). Detection of extended-spectrum beta-lactamase (ESBL)-producing Escherichia coli on flies at poultry farms. Applied and Environmental Microbiology 80(1):239-246.

Bogaerts P,Huang TD, Bouchahrouf W, Bauraing C, Berhin C,El Garch F, Glupczynski Y, ComPath Study Group (2015). Characterization of ESBL- and AmpC- producing Enterobacteriaceae from diseased companion animals in Europe. Microbial Drug Resistance21(6):643650.

Carattoli A, Lovari S, Franco A, Cordaro G, Di Matteo P, Battisti A (2005). Extended-spectrum beta-lactamases in Escherichia coli isolated from dogs and cats in Rome, Italy, from 2001 to 2003. Antimicrobial Agents and Chemotherapy 49:833-835.

Carvalho AC, Barbosa AV, Araisb LR, Ribeirob PF, Carneiro VC, Cerqueira AMF (2016). Resistance patterns, ESBL genes, and genetic relatedness of Escherichia coli from dogs and owners. Brazilian Journal of Microbiology 47:150-158.

Chah KF, Nweze NE (2001). Antibiotic use in poultry production in Nsukka, Southeast Nigeria. Proceedings of Nigerian Society of Animal Production 26:69-72.

Chah KF, Oboegbulem SI (2007). Extended spectrum beta-lactamase production among ampicillin-resistant Escherichia coli strains from chicken in Enugu State, Nigeria. Brazilian Journal of Microbiology 38:681-686

Clinical and Laboratory Standards Institute (CLSI) (2012). Performance standards for antimicrobial susceptibility testing; twenty-second informational supplement. M100-S2232(3):62-78.

Clinical and Laboratory Standards Institute (CLSI) (2014). Performance standards for antimicrobial susceptibility testing, twenty-fourth informational supplement, M100-S2434(1):61-188.

Coque TM, Baquero F, Canton R (2008). Increasing prevalence of ESBL producing Enterobacteriaceae in Europe. European Surveill p 13.

Costa D, Poeta P, Briñas L, Sáenz Y, Rodrigues J, Torres C (2004). Detection of CTX-M-1 and TEM-52 beta-lactamases in Escherichia coli strains from healthy pets in Portugal. Journal of Antimicrobial Chemotherapy 54:960-961.

Costa D, Poeta P, SaenzY, Coelho, AC, Matos M, Vinue L, Rodrigues J, Torres C (2008). Prevalence of antimicrobial resistance and resistance genes in faecal Escherichia coli isolates recovered from healthy pets. Veterinary Microbiology 127:97-105.
Cozma A, Carp-Carare C, Porea D, Mares M, Guguianu E, Rimbu C, Carp-Carare M (2015b). The prevalence of ESBL-producing strains of E. coli and K. pneumoniae, isolated from pets treated with antibiotics preliminary remarks. Bulletin UASVM Veterinary Medicine 72(2):406-410.

Cozma AP,Carp-Cărare M,Ciocan OA,Carp-Cărare C, Guguianu E, Rîmbu C,Mares M (2015a). The prevalence of positive ESBLE. coli and Klebsiella pneumoniaestrains in the dogs in the Iași paddock. Lucrari Stiintifice-Universitatea de Stiinte Agricole a Banatului Timisoara, Medicina Veterinara 48(2):74-80.

Damborg P, Gaustad IB, Olsen JE, Guardabassi L (2011). Selection of CMY-2 producing Escherichia coli in the faecal flora of dogs treated with cephalexin. Veterinary Microbiology 151(3-4):404-408.

Damborg P, Morsing MK, Petersen T, Bortolaia V, Guardabassi L (2015) CTX-M-1 and CTX-M-15-producing Escherichia coli in dog faeces from public gardens. Acta Veterinaa Scandinavica 571:83.

Dierikx CM, van Duijkeren E, Schoormans AHW, van Essen-Zandbergen A, Veldman K, Kant A, Huijsdens XW, van der Zwaluw K, Wagenaar JA, Mevius DJ (2012). Occurrence and characteristics of extendedspectrum- $\beta$-lactamase and AmpC-producing clinical isolates derived from companion animals and horses. Journal of Antimicrobial Chemotherapy doi:10.1093/jac/dks049.

Duru C, Nwanegbo E, Adikwu M, Ejikeugwu C, Esimone C (2013). Extended-spectrum beta-lactamase-producing Escherichia coli strains of poultry origin in Owerri, Nigeria. World Journal of Medical Sciences $8(4): 349-354$.

Dwarz SM, Bonomo RA (2010). Three decades of beta-lactamase inhibitors. Clinical Microbiological Reviews 23(1):160-201.

Ewers C, Grobbel M, Bethe A, Wieler LH, Guenther S (2011). Extendedspectrum beta-lactamases-producing gram-negative bacteria in companion animals: action is clearly warranted! Berl Munch Tierarztl Wochenschr 124:94101.

Ewers C, Bethe A, Semmler T, Guenther S, Wieler LH (2012). Extendedspectrum $\beta$-lactamase-producing and AmpC-producing Escherichia coli from livestock and companion animals, and their putative impact on public health: a global perspective. Clinical Microbiology Infection 18:646-655.

Ewers C, Grobbel M, Stamm I, Kopp PA, Diehl I, Semmler T, Fruth A, Beutlich J, Guerra B, Wieler LH, Guenther S (2010). Emergence of human pandemic O25:H4ST131 CTX-M-15 extended-spectrumbetalactamase-producing Escherichia coli among companion animals. Journal of Antimicrobial Chemotherapy 65:651-660.

Ewers C,Bethe A,Stamm I,Grobbel M,Kopp PA, Guerra B,Stubbe M,Doi Y,Zong Z, Kola A, Schaufler K, Semmler T, Fruth A, Wieler LH, Guenther S (2014). CTX-M-15-D-ST648 Escherichia coli from companion animals and horses: another pandemic clone combining multiresistance and extraintestinal virulence? Journal of Antimicrobial Chemotherapy 69(5):12241230.

Eze E, Nwakeze E, Oji A, Ejikeugwu C, Iroha I (2013). Microbiological investigation of Escherichia coli isolates from cloacal and feacal swabs of broiler chickens for extended- spectrum beta-lactamase (ESBL) enzymes. Journal of Pharmacy and Biological Sciences 7(5):96-99. 
Febler AT, Kadlec K, Hassel M, Hauschild T, Eidam C, Ehricht R, Monecke S, Schwarz S (2011). Characterization of methicillin resistant Staphylococcus aureus isolates from food and food products of poultry origin in Germany. Applied and Environmental Microbiology $77(20): 7151-7157$.

Feria C, Ferreira E, Correia JD, Goncalves J, Canica M (2002). Patterns and mechanisms of resistance to $\beta$-lactams and $\beta$-lactamase inhibitors in uropathogenic Escherichia coli isolated from dogs in Portugal. Journal of Antimicrobial Chemotherapy 49:77-85.

Filipovic I, Masic D, Asanin R (2007). Investigation of the presence extended spectrum beta-lactamase (ESBL) in multiresistant strains of E.coli and Salmonella species originated from domestic animals. Acta Veterinaria (Beograd) 57(4):369-379.

Frank T, Mbecko JR, Misatou P, Monchy D (2011). Emergence of quinolone resistance among extended-spectrum beta-lactamase producing Enterobacteriaceae in the Central African Republic: genetic characterization. BioMed Central Research Notes 4:309.

Gandolfi-Decristophoris P,Petrini O,Ruggeri-Bernardi N,Schelling E (2013). Extended-spectrum $\beta$-lactamase-producing Enterobacteriaceat in healthy companion animals living in nursing homes and in the community. American Journal of Infection Control 41(9):831-835.

Gibson JS, Cobbold RN, Trott DJ (2010). Characterization of multidrugresistant Escherichia coli isolated from extraintestinal clinical infections in animals. Journal of Medical Microbiology 59:592-598.

Guerra B, FischerJ,Helmuth R(2014). An emerging publichealth problem: acquired carbapenemase-producing microorganisms are present in foodproducing animals, their environment, companion animals and wild birds. Veterinary Microbiology 171(3-4):290-297.

Guardabassi L, Schwarz S, Lloyd DH (2004). Pet animals as reservoirs of antimicrobial-resistant bacteria. Antimicrobial Agents and Chemotherapy 54:321-332.

Harada K, Niina A, Shimizu T, Mukai Y, Kuwajima K, Miyamoto T, Kataoka Y (2014). Phenotypic and molecular characterization of antimicrobial resistance in Proteus mirabilis isolates from dogs. Journal of Medical Microbiology 63:1561-1567.

Harada K,Niina A,Nakai Y, Kataoka Y, Takahashi T (2012). Prevalence of antimicrobial resistance in relation to virulence genes and phylogenetic origins among urogenital Escherichia coli isolates from dogs and cats in Japan. American Journal of Veterinary Research 73(3):409-417.

Hordijk J, Schoormans A, Kwakernaak M, Duim B, Broens E, Dierikx C, Mevius D, J Wagenaar (2013). High prevalence of fecal carriage of extended-spectrum $\beta$-lactamase/AmpC-producing Enterobacteriaceat in cats and dogs. Frontiers in Microbiology 4:1-5.

Huber H, Zweifel C, Wittenbrink MM, Roger S (2013). ESBL-producing uropathogenic Escherichia coli isolated from dogs and cats in Switzerland. Veterinary Microbiology 162:992-996.

Jacoby GA (2005). Mechanism of resistance to quinolones. Clinical Infectious Diseases 41 (Supplement 2):S120-S126.doi:10.1086/ 428052 .

Inwezerua $\mathrm{C}$, Mendonça N, Calhau V, Domingues S, Adeleke OE, DaSilva GJ (2014). Occurrence of extended-spectrum beta-lactamases in human and bovine isolates of Escherichia coli from Oyo State, Nigeria. Journal of Infection in Developing Countries 8(6):774779.
Johard S, Börjesson S, Trowald-Wigh G, Fernström L, Nicol C, Bergström A (2015). Extended spectrum beta-lactamase/AmpC-producing E. coli in dogs treated with antimicrobials in surgical wards. International Journal of Applied Research in Veterinary Medicine 13(1):80-88.

Leclercq R, Canton R, Brown DFJ, Giske CG, Heisig P, MacGowan AP, Mouton JW, Nordmann P, Rodloff AC, Rossolini GM, Soussy CJ, Steinbakk M, Winstanley TG, Kahlmeter G (2013). EUCAST expert rules in antimicrobial susceptibility testing. Clinical Microbiology and Infection 19:141-160.

Lim SK, Lee HS, Nam HM, Jung SC, Bae YC (2009). CTX-M-type betalactamase in Escherichia coli isolated from sick animals in Korea. Microbial Drug Resistance 15(2):139-142.

Ljungquist O, Ljungquist D, Myrena M, Ryden C, Finn M, Bengtsson B (2016). Evidence of household transfer of ESBL-/pAmpCproducing Enterobacteriaceae between humans and dogs a pilot study. Infection Ecology and Epidemiology 6: 31514 http://dx.doi.org/10.3402/ iee.v 6.31514

Ma J,ZengZ, Chen Z, Xu X, Wang X, Deng Y, Lu D, Huang L, Zhang Y, Liu J, Wang M (2009). High prevalence of plasmid-mediated quinolone resistance determinants qnr, aac (6_-Ib-cr, and qep $A$ among ceftiofur-resistant Enterobacteriaceae isolates from companion and foodproducing animals. Antimicrobial Agents and Chemotherapy 53(2):519-524.

Magiorakos AP, Srinivasan A, Carey RB, Carmeli Y,Falagas ME, Giske CG,Harbarth S,Hindler JF,Kahlmeter G,Olsson-Liljequist B, Paterson DL, Rice LB, Stelling J, Struelens MJ, Vatopoulos A, Weber JT, Monnet DL (2011). Multidrug-resistant, extensively drug-resistant and pandrug-resistant bacteria: an international expert proposal for interim standard definitions for acquired resistance. Clinical Microbiology and Infection doi:10.1111/j.1469-0691.2011.03570.x.

Matsumoto Y, Ikeda F, Kamimura T, Yokota Y, Mine Y (1988). Novel plasmid-mediated B-lactamase from Escherichia coli that inactivates oxyimino-cephalosporins. Antimicrobial Agents and Chemotherapy 32:1243-1246.

Meirelles-Pereira F, de Meirelles Santos Pereira A, da Silva MCG, Gonçalves VD, Brum PR, de Castro EAR, PereiraAA, de Assis Esteves F, Pereira JAA (2002). Ecological aspects of the antimicrobial resistance in bacteria of importance to human infections. Brazilian Journal of Microbiology 33:287-293.

Meyer E, Gastmeier P, Kola A,Schwab F. (2012). Pet animals and foreign travel are risk factors for colonisation with extended-spectrum $\beta$ lactamase-producing Escherichia coli.Infection 40(6):685-687.

Moreno A,Bello H,Guggiana D,Domínguez M,González G (2008). Extended-spectrum beta-lactamases belonging to CTX-M group produced by Escherichia coli strains isolated from companion animals treated with enrofloxacin. Veterinary Microbiology 129(1-2):203-208.

Morosini MI, Garcia-Castillo M, Coque TM, Valverde A, Novai SA, Loza E, Baqquero F, Canton R (2006). Antibiotic coresistance in extendedspectrum $\beta$-lactamase-producing Enterobacteriaceae and in vitro activity of tigecycline. Antimicrobial Agents and Chemotherapy 50(8):26952699.

Morrison BJ, Rubin JE (2015). Carbapenemase producing bacteria in the food supply escaping detection. PLOS ONE doi:10.1371/journal. 
32 pone.0126717.

Murphy C, Reid-Smith RJ, Prescott, JF, Bonnett BN, Poppe C, Boerlin P, Weese JS, Janecko N,McEwen SA (2009). Occurrence of antimicrobial resistant bacteria in healthy dogs and cats presented to private veterinary hospitals in southern Ontario: a preliminary study. Canadian Veterinary Journal 50:1047-1053.

Murphy CP, Reid-Smith RJ, Boerlin P, Weese JS, Prescott JF, Janecko N,Hassard L, McEwen SA (2010). Escherichia coli and selected veterinary and zoonotic pathogens isolated from environmental sites in companion animal veterinary hospitals in southern Ontario. Canadian Veterinary Journal 51:963-972

Nam HM, Lee HS, Byun JW, Yoon SS, Jung SC,Joo YS, Lim SK (2010). Prevalence of antimicrobial resistance in fecal Escherichia coli isolates from stray pet dogs and hospitalized pet dogs in Korea. Microbial Drug Resistance 16(1):75-79.

Nebbia P, Tramuta C,Odore R,Nucera D,Zanatta R, Robino P (2014). Genetic and phenotypic characterisation of Escherichia coli producing cefotaximase-type extended-spectrum $\beta$-lactamases: first evidence of the ST131 clone in cats with urinary infections in Italy. Journal of Feline Medicine and Surgery 16(12):966-971.

Nilsson O (2015). Hygiene quality and presence of ESBL-producing Escherichia coli in raw food diets for dogs. Infection Ecology and Epidemiology 5:28758doi:http://dx.doi.org/10.3402/iee.v5.28758

O'Keefe A, Hutton TA, Schifferli DM, Rankin SC (2010). First detection ofCTX-M andSHV extended-spectrum beta-lactamases in Escherichia coli urinary tract isolates from dogs and cats in the United States. Antimicrobial Agents and Chemotherapy 54:3489-3492.

Oliveira PA, Moura RA, Rodrigues GV, Lopes KFC, Zaniolo MM, Rubio KAJ, Dias EH, Oliveira LA, Chideroli RT, Gonçalves DD (2016). Detection of extended spectrum beta-lactamases and resistance in members of the Enterobacteriaceae family isolated from healthy sheep and dogs in Umuarama, Paraná, Brazil. Sciências Agrárias, Londrina 37(2):829-840.

Olowe OA, Adewumi O, Odewale G, Ojurongbe O, Adefioye OJOA (2015). Phenotypic and molecular Characterisation of extendedspectrum beta-lactamase producing Escherichia coli obtained from animal fecal samples in Ado Ekiti, Nigeria. Journal of Environmental and Public Health http://dx.doi.org/10.1155/2015/49798.

Pfeifer Y, Cullik A, Witte W (2010). Resistance to cephalosporins and carbapenems in Gram-negative bacterial pathogens. International Journal of Medical Microbiology 300:371-379.

Poirel L, Nordmann P, Ducroz S, Boulouis HJ, Arne P, Millemann Y (2013). Extended-spectrum beta-lactamase CTX-M-15-producing Klebsiella pneumoniae of sequence type ST274 in companion animals. Antimicrobial Agents and Chemotherapy 57:2372-2375.

Pomba C, da Fonseca JD, Baptista BC, CorreiaJD (2009) Detection of the pandemic O25-ST131 human virulent Escherichia coli CTX-M-15producing clone harboring the qnrB2 and aac (6_)-Ib-cr genes in a dog. Antimicrobial Agents and Chemotherapy 53(1):327-328.

Rocha-Gracia RC, Cortes-Cortes G, Lozano-Zarain P, Bello F, MartinezLaguna Y, Torres C (2015). Faecal Escherichia coli isolates from healthy dogs harbour CTX-M-15 and CMY-2 betalactamases. Veterinary
Journal 203(3):315-319.

Rubin JE, Pitout JD (2014). Extended-spectrum $\beta$-lactamase, carbapenemase and AmpC producing Enterobacteriaceae in companion animals. Veterinary Microbiology 170:10-18.

Rzewuska M, Czopowicz MB, Kizerwetter-Uwida M, Chrobak D, Baszczak BB, Binek M (2015). Multidrug Resistance in Escherichia coli strains isolated from infections in dogs and cats in Poland (2007-2013). The Scientific World Journal http://dx.doi.org/10.1155/2015 1408205 .

Schaufler K, Bethe A, Lubke-Becker A, Ewers C, Kohn B, Wieler LH, Guenther S (2015). Putative connection between zoonotic multiresistant extended-spectrum beta-lactamase (ESBL)-producing Escherichia coli in dog feces from a veterinary campus and clinical isolates from dogs. Infection Ecology and Epidemiology 5:25334 doi: http://dx.doi.org/10.3402/iee.v5.25334.

Schmiedel J, Falgenhauer L, Domann E, Bauerfeind R, Prenger-Berninghoff E, Imirzalioglu C, Trinad Chakraborty (2014). Multiresistant extendedspectrum $\beta$-lactamase producing Enterobacteriaceae from humans, companion animals and horses in central Hesse, Germany. BMC Microbiology 14:187.

Shaheen BW, Nayak R, Boothe DM (2013). Emergence of a New Delhi metallo-- $\beta$-lactamase (NDM-1)-encoding gene in clinical Escherichia coli isolates recovered from companion animals in the United States. Antimicrobial Agents and Chemotherapy 57:2902-2903.

Shaheen BW, Nayak R, Foley SL, Kweon O, Deck J, Park M, Rafii F, Boothe DM (2011). Molecular characterization of resistance to extended spectrum cephalosporins in clinical Escherichia coli isolates from companion animals in the United States. Antimicrobial Agents and Chemotherapy 55:5666-5675.

Sidjabat HE, Hanson ND, Smith-Moland E, Bell JM, Gibson JS, Filippich LJ, Trott DJ (2007). Identification of plasmid-mediated extended spectrum and AmpC $\beta$-lactamases in Enterobacter spp. isolated from dogs. Journal of Medical Microbiology 56:426-434.

So JH, Kim J,Bae IK, Jeong SH, Kim SH,Lim SK, Park YH,Lee K (2012). Dissemination of multidrug-resistant Escherichia coli in Korean veterinary hospitals. Diagnostic Microbiology and Infectious Disease 73(2):195-199.

Srisanga S,Angkititrakul S, Sringam P,Ho PT,Vo AT, Chuanchuen R (2016). Phenotypic and genotypic antimicrobial resistance and virulence genes of Salmonella enterica isolated from pet dogs and cats. Journal of Veterinary Science [Epubahead of print].

Swedish Veterinary Antimicrobial Resistance Monitoring (SVARM) (2011). Swedish Veterinary Antimicrobial Resistance Monitoring. http://www.sva.se/globalassets/redesign2011/pdf/om_sva/publikation er/trycksaker/svarm2011.pdf.

Sun Y, Zeng Z, Chen S, Ma J, He L, Liu Y, Deng Y, Lei T, Zhao J, Liu JH (2010). High prevalence of blaCTX-M extended-spectrum $\beta$-lactamase genes in Escherichia coli isolates from pets and emergence of CTX-M-64 in China. Clinical Microbiology and Infection 16:1475-1481.

TamangMD, Nam HM, Jang GC, Kim SR, Chae MH,JungS-K, Byun JW,Park YH, and Suk-Kyung Lim (2012). Molecular characterization of extended-spectrum-beta-lactamase-producing and plasmid-mediated AmpC beta-lactamase-producing Escherichia coli isolated from stray 
33

dogs in South Korea. Antimicrobial Agents and Chemotherapy 56(5):2705-2712.

TimofteD,Dandrieux J, WattretA, FickJ, Williams J(2011). Detection of extended-spectrum- $\beta$-lactamase-positive Escherichia coli in bile isolates from two dogs with bacterial cholangiohepatitis. Journal of Clinical Microbiology 49(9):3411-3414.

Timofte D, Maciuca IE, Williams NJ, Wattret A, Schmidt V (2016). Veterinary hospital dissemination of CTX-M-15 extended-spectrum beta-lactamase-producing Escherichia coli ST410 in the United Kingdom. Microbial Drug Resistance 22(7):609-615.

Torkan S, Bahadoranian MA, Khamesipour F, Anyanwu MU (2016). Detection of virulence and antimicrobial resistance genes in Escherichia coli isolates from diarrhoiec dogs in Iran. Archivos de Medicina Veterinaria 48:181-190.

Torkan S, Khamesipour F, Anyanwu MU (2015). Detection of virulence and antibacterial resistance genes in Salmonella isolates from diarrhoeic dogs in Iran. Revue Médicine Véterinaire 166:221-228.

Torres C, Ugwu IC, Okpala A, Ceballos S, Alonso CA, Nwanta JN, Chah KF (2015). Occurrence and characterization of extended-spectrumbeta-lactamase producing enteric bacteria from market chickens in Nsukka, Nigeria. https://www.escmid.org/escmid_publications/ escmid_elibrary/material/?m.

Tuerena I, Williams NJ, Nuttall T, Pinchbeck G (2016). Antimicrobialresistant Escherichia coli in hospitalised companion animals and their hospital environment. Journal ofSmall Animal Practice 57:339-347.

Ugwu IC, Anyanwu MU, Ugwu CC, Ugwuanyi OW (2015). Prevalence and antibiogram of generic extended-spectrum beta-lactam-resistant enterobacteria in healthy pigs. Notulae Scientia Biologicae 7(3):272-280.

Urmunova V (2015). Extended spectrum beta-lactamase producing animal enterobacteriaceae isolates as potential risk to public health. Revue Medicine Veterinaire 166:7-8:192-207. van der Bij AK, Pitout JDD ( 2012). The role of international travel in the worldwide spread of multiresistant Enterobacteriaceae Journal of Antimicrobial Chemotherapy doi:10.1093/jac/dks214.

Wieler LH,Ewers C, Guenther S, Walther B, Lübke-Becker A (2011). Methicillin-resistant staphylococci (MRS) and extended-spectrum betalactamases (ESBL)-producing Enterobacteriaceae in companion animals: nosocomial infections as one reason for the rising prevalence of these potential zoonotic pathogens in clinical samples. International Journal of Medical Microbiology 301(8):635-641.

Woerther P, Burdet C, Chachaty E, Andremont A (2013). Trends in human fecal Carriage of Extended-spectrum- - -lactamases in the community: toward the globalization of CTX-M. Clinical Microbiology Reviews 26(4):744758.

World Health Organization (WHO) (2014). Antimicrobial resistance: global report on surveillance. World Health Organization.

World Health Organisation (WHO) (2011). Critically Important Antimicrobials for human medicine 3rd Revision. WHO, Geneva, Switzerland.

Yousfi M, Mairi A, Bakour S, Touati A, Hassissen L, Hadjadj L, Rolain J-M (2015). First report of NDM-5-producing Escherichia coli ST1284 isolated from dog in Bejaia, Algeria. New Microbes and New Infection 8:17-18.

Yousfi M, Touati A,Mairi A,Brasme L, Gharout-Sait A, Guillard T,De Champs C (2016). Emergence of carbapenemase-producing Escherichia coli isolated from companion Animals in Algeria. Microbial DrugResistance 22(4):342-346. 